\title{
Comparative Analysis of Deoxynivalenol Biosynthesis Related Gene Expression among Different Chemotypes of Fusarium graminearum in Spring Wheat
}

\author{
Chami C. Amarasinghe and W. G. Dilantha Fernando* \\ Department of Plant Science, University of Manitoba, Winnipeg, MB, Canada
}

Fusarium mycotoxins, deoxynivalenol (DON) and nivalenol (NIV) act as virulence factors and are essential for symptom development after initial infection in wheat. To date, 16 genes have been identified in the DON biosynthesis pathway. However, a

OPEN ACCESS

Edited by:

Daniela Gwiazdowska,

Poznań University of Economics,

Poland

Reviewed by:

Sean Doyle,

Maynooth University, Ireland

Friday Obanor,

Commonwealth Scientific

and Industrial Research Organisation

Australia

*Correspondence:

W. G. Dilantha Fernando

dilantha.fernando@umanitoba.ca

Specialty section:

This article was submitted to

Fungi and Their Interactions,

a section of the journal

Frontiers in Microbiology

Received: 14 April 2016

Accepted: 25 July 2016

Published: 08 August 2016

Citation:

Amarasinghe CC and

Fernando WGD (2016) Comparative

Analysis of Deoxynivalenol

Biosynthesis Related Gene

Expression among Different

Chemotypes of Fusarium

graminearum in Spring Wheat.

Front. Microbiol. 7:1229.

doi: 10.3389/fmicb.2016.01229 comparative gene expression analysis in different chemotypes of Fusarium graminearum in response to Fusarium head blight infection remains to be explored. Therefore, in this study, nine genes that involved in trichothecene biosynthesis were analyzed among 3-acetyldeoxynivalenol (3-ADON), 15-acetyldeoxynivalenol (15-ADON) and nivalenol producing F. graminearum strains in a time course study. Quantitative reverse transcription polymerase chain reaction revealed that the expression of all examined TRI gene transcripts initiated at 2 days post-inoculation (dpi), peaked at three to four dpi and gradually decreased at seven dpi. The early induction of TRI genes indicates that presence of high levels of $T R I$ gene transcripts at early stages is important to initiate the biosynthetic pathway of DON and NIV. Comparison of gene expression among the three chemotypes showed that relative expression of TR/ genes was higher in 3-ADON producing strains compared with 15-ADON and NIV strains. Comparatively higher levels of gene expression may contribute to the higher levels of DON produced by 3-ADON strains in infected grains.

Keywords: Fusarium graminearum, wheat, trichothecenes, chemotype, qRT-PCR, TRI genes

\section{INTRODUCTION}

Fusarium head blight (FHB) is one of the major economically important fungal diseases in wheat, barley, corn, and other small grains worldwide. Wheat yield losses of up to $50 \%$ have been reported in North America due to FHB (McMullen et al., 1997; Goswami and Kistler, 2005). One of the major concerns of FHB is the contamination of infected grains with Fusarium mycotoxins. Fusarium mycotoxins represent the largest group of mycotoxins, which contains more than 140 known metabolites such as trichothecenes, zearalenone and fumonisins (Yazar and Omurtag, 2008; Sobrova et al., 2010). Among these mycotoxins, trichothecenes are one of the major Fusarium mycotoxins synthesized mainly by the members in the Fusarium graminearum species complex (FGSC), F. culmorum, F. sprotrichioides and F. poae (Desjardins et al., 1993; Foroud and Eudes, 2009; Wang et al., 2011). The fungi in the FGSC have the potential to devastate 
a crop by reducing grain quality and quantity. After Fusarium infection, the grains become contaminated with trichothecene mycotoxins such as deoxynivalenol (DON), produced by the pathogen, making the crop unsuitable for food and feed. Trichothecenes produced by Fusarium spp. act as virulence factors in wheat plants. It has been reported that DON is important for the spread of F. graminearum beyond the point of infection within the host plant. Proctor et al. (1995) have shown that TRI5 ${ }^{-}$mutants have reduced virulence compared to wild type strains in Wheat and Rye cultivars suggesting that trichothecene production contributes to the virulence of $F$. graminearum. Non-DON producing strains of $F$. graminearum can initiate the infection, but not spread within the host tissue (Proctor et al., 1995; Bai et al., 2002). A study done by Diamond et al. (2013) found that DON is capable of inhibiting the apoptosislike programmed cell death in Arabidopsis cell cultures subjected to heat stress.

So far, 16 genes have been characterized in the DON biosynthesis pathway. These genes reside at four different loci on different chromosomes; the core TRI cluster consists of 12 genes located on chromosome 2, the TRI1-TRI16 loci on chromosome 1, TRI101 on chromosome 4, and TRI15 on chromosome 3, respectively (Gale et al., 2005; Alexander et al., 2009; Merhej et al., 2011). The first step in the DON biosynthesis pathway consists of the cyclization of the initial substrate, farnesyl pyrophosphate (FPP) to produce non-toxic trichodiene, by the trichodiene synthase enzyme encoded by TRI5 gene (Hohn and Beremand, 1989). The next nine reactions in the pathway are mediated by the enzymes encoded by TRI4, TRI101, TRI11 and TRI3, respectively. These reactions lead to the formation of calonectrin, which serves as a substrate for the production of 3-ADON, 15-ADON and 4-acetylnivalenol (4-ANIV) (Alexander et al., 2009; Foroud and Eudes, 2009; Merhej et al., 2011). The genes TRI7 and TRI13 are functional only in F. graminearum strains that are capable of producing NIV (Brown et al., 2001; Lee et al., 2002). The enzymes encoded by TRI7 and TRI13 genes mediate two common steps following calonectrin. In nivalenol producing $F$. graminearum strains, the pathway continues with the product of TRI1 to produce 4-ANIV and the final step mediated by TRI8 to give NIV. The TRI7 and TRI13 genes are not active in DON producers; therefore, DON biosynthesis proceeds directly from calonectrin with the enzymes encoded by TRI1 gene (McCormick and Alexander, 2002; Alexander et al., 2011; Merhej et al., 2011). The formation of $3-\mathrm{ADON}$ or $15-\mathrm{ADON}$ is strain specific and decided by the esterase coding sequence of TRI8 gene (Alexander et al., 2011). To date, limited research has been done on expression of TRI genes in different chemotypes of F. graminearum during wheat colonization.

Among the different TRI genes, TRI5 gene has received more attention and so far the majority of studies have focused on the expression of the TRI5 gene during Fusariumwheat colonization. A study done by Hallen-Adams et al. (2011) examined the expression of the TRI5 gene during wheat spike infection of susceptible and resistant cultivars and susceptible cultivars treated with strobilurin fungicides. The highest expression of the TRI5 gene was observed at the infection front. Gardiner et al. (2009) reported that TRI5 gene is strongly expressed in the rachis tissue of wheat. In this study they used a $F$. graminearum strain constructed by fusing a green fluorescent protein (GFP) marker to the promoter of TRI5 gene. Zhang et al. (2009) examined the expression of the TRI5 gene between carbendazim-resistant and sensitive F. graminearum in shake culture and reported a significant exponential relationship between trichothecene production and TRI5 gene expression. More recently Lee et al. (2014) compared the expression of TRI cluster genes in DON vs. NIV producing F. graminearum strains in liquid cultures. No study has been done to compare the level of expression of TRI genes in different chemotypes of $F$. graminearum during wheat colonization.

Therefore, in this study we have compared the level of expression of nine TRI genes in 3-ADON, 15-ADON and NIVproducing $F$. graminearum strains in a time course study both in resistant and susceptible wheat cultivars. The objective of this study was to evaluate the chemotype specific gene expression patterns in trichothecene biosynthesis related genes in different chemotypes of F. graminearum during wheat infection and colonization.

\section{MATERIALS AND METHODS}

\section{Greenhouse Experiment and RNA Isolation}

Two wheat cultivars with different levels of resistance to Fusarium head blight (FHB) were used in this study. A spring wheat cultivar, Roblin, which is highly susceptible (S) to FHB, and a FHB moderately resistant (MR) cultivar, Carberry, with resistance originating from the Chinese cultivar Sumai3 were used in the study. The Chinese cultivar Sumai3 have both Type I and II FHB resistance (Bai and Shaner, 1994). To prepare inoculum, two F. graminearum strains from each chemotype were cultured on Spezieller Nährstoffarmer agar (SNA) medium (0.2 g glucose, $0.2 \mathrm{~g}$ sucrose, $1 \mathrm{~g} \mathrm{KH}_{2} \mathrm{PO}_{4}, 1 \mathrm{~g} \mathrm{KNO}_{3}, 0.25 \mathrm{~g}$ $\mathrm{MgSO}_{4} .7 \mathrm{H}_{2} \mathrm{O}, 0.5 \mathrm{~g} \mathrm{KCl}, 14 \mathrm{~g}$ technical agar in $1 \mathrm{~L}$ of distilled water). F. graminearum strains used in this study were consisted of; Q-06-11 (designated as: 3-ADON1, isolated from wheat in Canada), A6-06-01 (3-ADON2, isolated from wheat in Canada), PH1 (15-ADON1, isolated from wheat in the USA), M2-06-02 (15-ADON2, isolated from wheat in Canada), W52516 (NIV1, isolated from maize in China) and W56604 (NIV2, isolated from maize in China). To produce liquid inoculum, $1.5 \mathrm{~L}$ of carboxymethyl cellulose (CMC) liquid media (15 g CMC, $1 \mathrm{~g}$ $\mathrm{NH}_{4} \mathrm{NO}_{3}, 1 \mathrm{~g} \mathrm{KH}_{2} \mathrm{PO}_{4}$ monobasic, 5 g MgSO $4.7 \mathrm{H}_{2} \mathrm{O}$, 1 g yeast extract in $1 \mathrm{~L}$ of distilled water) was prepared and four SNA media (Leslie and Summerell, 2006) plates from each strain were divided into sections and added into each flask. Seven days after incubation at $25^{\circ} \mathrm{C}$ under fluorescent light, the number of conidia per milliliter was determined by using a haemocytometer. The final conidial concentration was adjusted to 50,000 conidia/mL using distilled water. Seeds of spring wheat cultivars; Carberry and Roblin were planted in $15-\mathrm{cm}$ plastic pots and maintained at $22-24^{\circ} \mathrm{C}$ in the greenhouse at the Department of Plant Science, University of Manitoba, Winnipeg, MB, Canada. Inoculations were conducted at $30-50 \%$ anthesis. A $10 \mu \mathrm{L}$ of F. graminearum 
suspension $(50,000$ conidia/mL) was injected between the palea and lemma of spikelets per each spike according to the protocol described by Cuthbert et al. (2006). Five biological replicates for each strain and time point were conducted following a complete randomized design. Four to five spikes were inoculated per plant. FHB disease severity (DS) ratings were taken at 2, $3,4,7,10$, and 14 days post-inoculation (dpi) using the FHB disease scale by Stack and McMullen (1995). FHB DS readings were taken from five inoculated spikes for each replicate. The inoculated spikes were sampled at 2, 3, 4, 7, 10, and $14 \mathrm{dpi}$ and stored at $-80^{\circ} \mathrm{C}$ freezer until RNA isolation. The mock inoculations were made using distilled water in both Roblin and Carberry for all time points. The inoculated spikes from five replicates were pooled and ground into fine powder in liquid nitrogen using a mortar and pestle. Total RNA was isolated using TRIzol $^{\circledR}$ reagent (Invitrogen Life Technologies, Carlsbad, CA, USA) according to manufacturer's instructions. Extracted RNA was quantified using the NanoDrop 3300 (Thermo Scientific Inc., Wilmington, DE, USA). The integrity of RNA was analyzed using $1 \%$ agarose gel electrophoresis. To remove any DNA contaminations, RNA was treated with TURBO $^{\mathrm{TM}}$ DNaseI (Invitrogen Life Technologies, Carlsbad, CA, USA) before cDNA synthesis. The first strand of cDNA was synthesized from $2 \mu \mathrm{g}$ total RNA as the template using SuperScript ${ }^{\mathrm{TM}}$ III First-Strand Synthesis System for reverse transcription-polymerase chain reaction (Invitrogen Life Technologies, Carlsbad, CA, USA).

\section{FDK, DON and NIV Analysis}

Kernels were harvested from inoculated spikes from both cultivars Carberry and Roblin, at $14 \mathrm{dpi}$. The percentage of Fusarium damaged kernels (FDK) was estimated by taking a pooled sample of $10 \mathrm{~g}$ from all replicates. The same kernels used for FDK analysis were used for DON or NIV analysis. Wheat kernels of each strain were pooled, ground and analyzed by $\operatorname{Veratox}^{\circledR}$ DON 5/5 kit (product no: 8331, Neogen Corp., Lansing, MI, USA) for DON analysis. NIV analysis was done using GC-MS according to the protocol described by Tittlemier et al. (2013).

\section{Quantitative Reverse Transcription PCR}

A total of nine genes (TRI4, TRI5, TRI6, TRI3, TRI8, TRI101, TRI9, TRI12 and FPP) in the DON biosynthetic pathway were examined using quantitative reverse transcription PCR (qRTPCR). The level of expression of each gene was analyzed using a set of gene specific primers as described by Lee et al. (2014). As a house-keeping gene, translation elongation factor 1 alpha (EF-1 $\alpha$ ) from F. graminearum was selected (Kim and Yun, 2011). qRT-PCR reactions were performed in a CFX96 Touch $^{\text {TM }}$ Real Time PCR Detection System (Bio-Rad, Hercules, CA, USA) according to the protocol described by Lee et al. (2014). The qRT-PCR reaction cycles were consisted of initial denaturation at $95^{\circ} \mathrm{C}$ for $3 \mathrm{~min}$, followed by 45 cycles at $95^{\circ} \mathrm{C}$ for $10 \mathrm{~s}$, $60^{\circ} \mathrm{C}$ for $20 \mathrm{~s}, 72^{\circ} \mathrm{C}$ for $20 \mathrm{~s}$, and finally $95^{\circ} \mathrm{C}$ for $10 \mathrm{~s}$ and $65^{\circ} \mathrm{C}$ for $5 \mathrm{~s}$. The qRT-PCR reaction mixture contained $10 \mu \mathrm{L}$ of $2 \times$ iQ SYBR ${ }^{\circledR}$ Green Supermix consisted of SYBR ${ }^{\circledR}$ Green I dye, $50 \mathrm{U} / \mathrm{ml} \mathrm{iTaq}^{\mathrm{TM}}$ DNA polymerase, $0.4 \mathrm{mM}$ each dNTPs, $6 \mathrm{mM} \mathrm{MgCl}_{2}, 40 \mathrm{mM}$ Tris- $\mathrm{HCl}(\mathrm{pH}$ 8.4), $100 \mathrm{mM} \mathrm{KCl}, 20 \mathrm{nM}$ fluorescein and stabilizers (Bio-Rad, Hercules, CA, USA), $0.5 \mu \mathrm{L}$ of each primer (10 pM), $1 \mu \mathrm{L}$ of template cDNA (10 ng), and RNase free water to a final volume of $20 \mu \mathrm{L}$. Quantification values were analyzed using the Bio-Rad CFX Manager v1.6, and the threshold cycle $(\mathrm{Ct})$ values were determined. In all reactions, a non-template control (NTC) was set up to avoid any DNA contaminations in the reaction mixtures. Each reaction sample was amplified three times and final Ct values were calculated as an average of three replicates. The relative transcript abundance of the target genes was determined by the Pfaffl method (Pfaffl et al., 2002). qRT-PCR primer amplification efficiency was determined using the Ct slope method. In this method, serial dilutions of the template were prepared and $\mathrm{Ct}$ values were determined. Then a standard curve was generated by plotting the $\mathrm{Ct}$ values against the log cDNA concentrations. The amplification efficiency $(\mathrm{E})$ of each primer was determined using the formula $\mathrm{E}=10^{-1 / \text { slope }}$. The percent amplification efficiency was determined using the formula $\% \mathrm{E}=(\mathrm{E}-1) * 100 \%$. The percent amplification efficiency of all genes were ranged between 95.6 and $101.3 \%$.

\section{Statistical Analysis}

Analysis of variance (ANOVA) for FHB DS at seven and 14 dpi was performed using the PROC Mixed procedure of SAS software (SAS version 9.3, SAS Institute Inc., Cary, NC, USA). Cultivar, strain and cultivar* ${ }^{\star}$ strain were considered as fixed effects. The Bonferroni method was used to compare statistically significant differences in least squares (LS) means of all variables. The type 3 test of fixed effects was determined and those with $p \leq 0.05$ were considered significant.

\section{RESULTS}

Nine genes from the F. graminearum trichothecene biosynthesis pathway, TRI4, TRI5, TRI6, TRI3, TRI8, TRI101, TRI9, TRI12 and FPP along with the housekeeping gene $E F-1 \alpha$, were selected for gene expression analysis. Each selected gene was analyzed by quantitative reverse transcription PCR to examine the changes in transcript levels at different time intervals post-inoculation. Accumulation of TRI gene transcripts initiated as early as $2 \mathrm{dpi}$ in most strains. Significant differences were observed for cultivar, strain, and dpi for all the analyzed genes. The qRT-PCR analysis showed that the FPP transcript accumulation initiated at $2 \mathrm{dpi}$, peaked at 3-4 dpi and rapidly decreased at $7 \mathrm{dpi}$ in MR cultivar Carberry (Figure 1A). A similar transcript accumulation pattern was observed in the susceptible (S) cultivar Roblin, however, at $10 \mathrm{dpi}$ there was a slight increase in transcript accumulation in 3-ADON1, 3-ADON2 and 15-ADON1 strains and then gradually decreased at $14 \mathrm{dpi}$ (Figure 1B). In both MR cultivar and S cultivar, the abundance of FPP transcripts was higher in 3-ADON producing $F$. graminearum strains than $15-\mathrm{ADON}$ and NIV producing strains at most time points.

The qRT-PCR analysis for TRI5 showed that, transcript accumulation initiated at $2 \mathrm{dpi}$ and peaked at $4 \mathrm{dpi}$ and rapidly decreased by day 7 . In contrast to the expression pattern of 


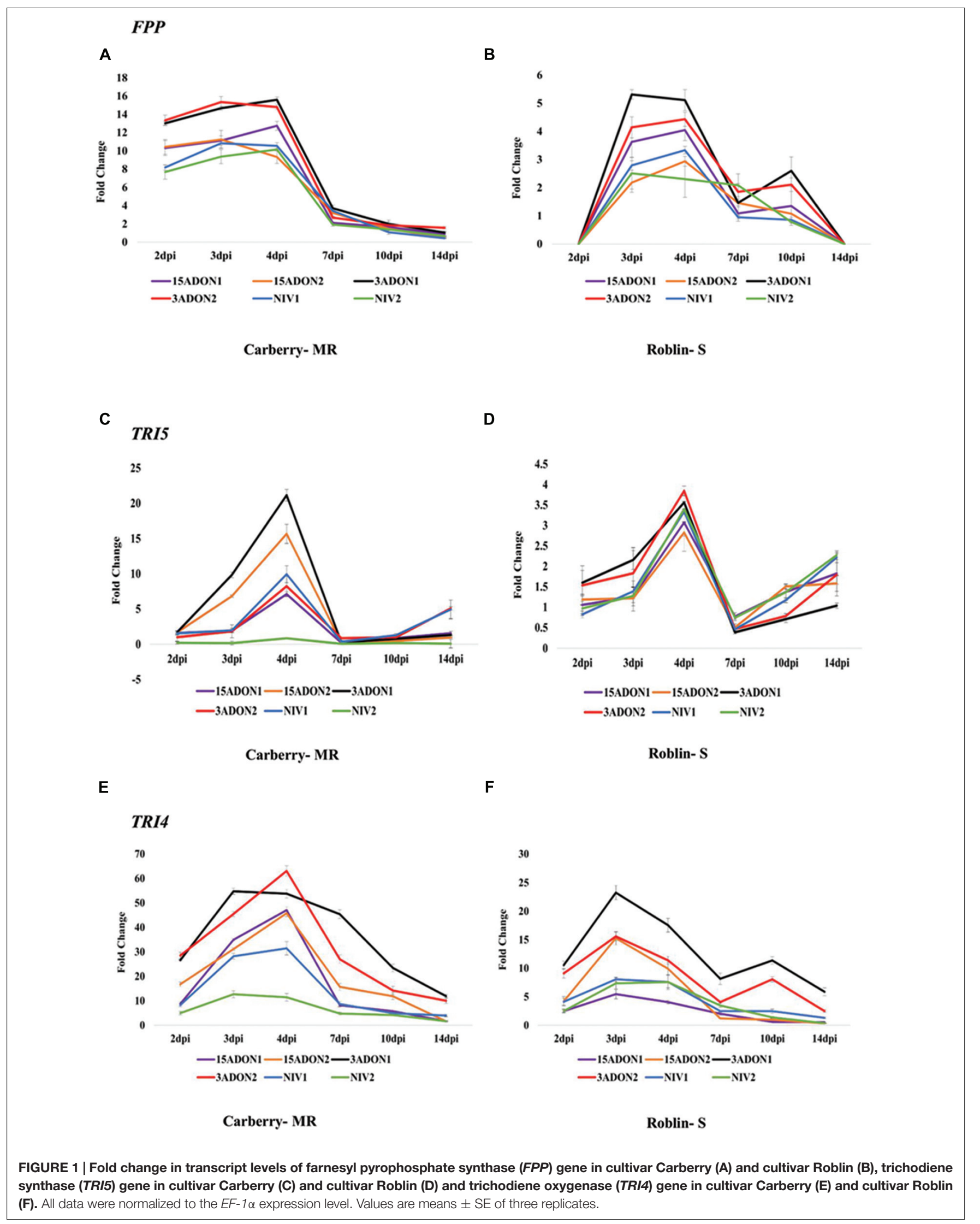


FPP gene, TRI5 gene expression increased again after 7 dpi in both cultivars (Figures 1C,D). Similar to FPP and TRI5 genes, accumulation of TRI4 transcripts initiated at $2 \mathrm{dpi}$, peaked at 3-4 dpi and started decreasing after day 4 in both cultivars (Figures 1E,F). However, in cultivar Roblin (S), transcript accumulation again peaked at $10 \mathrm{dpi}$ in 3 -ADON producing strains. The accumulation of TRI6 transcripts also initiated at $2 \mathrm{dpi}$, peaked at $4 \mathrm{dpi}$ and gradually decreased after day 4 in cultivar Carberry (MR) (Figure 2A). In cultivar Roblin (S), transcript accumulation initiated at $2 \mathrm{dpi}$, peaked at $3 \mathrm{dpi}$ and start decreasing after day 3 (Figure 2 B). In 3 -ADON producing strains the level of gene expression again peaked at $10 \mathrm{dpi}$. For TRI8 gene, transcript accumulation was initiated at $2 \mathrm{dpi}$ and peaked at $4 \mathrm{dpi}$ in all strains in cultivar Carberry (MR) (Figure 2C). In cultivar Roblin (S), transcript accumulation peaked at $3 \mathrm{dpi}$ in 3-ADON and 15-ADON strains whereas for NIV strains it was at 4 dpi (Figure 2D).

The expression of the TRI101 gene initiated at 2 dpi and remained relatively constant for 15-ADON1, 15-ADON2, NIV1 and NIV2 strains during the early time intervals at 2-7 dpi (no distinct peaks were observed) and started decreasing at $10 \mathrm{dpi}$ in MR cultivar, Carberry (Figure 2E). In 3-ADON1, peaks were observed at 4 and 10 dpi. A similar pattern was observed in $S$ cultivar Roblin, however, a distinct peak was observed for 3-ADON2 at 3 dpi (Figure 2F). Similar to other genes, the accumulation of TRI3 transcripts initiated at $2 \mathrm{dpi}$ and peaked at 3-4 dpi in most strains in both cultivars; however, the transcript abundance started decreasing at $4 \mathrm{dpi}$ (Figures 3A,B). Despite the earlier induction (2 dpi), the level of expression of TRI9 gene peaked at 7-10 dpi (in cultivar Carberry) and $10 \mathrm{dpi}$ (in cultivar Roblin) and gradually decreased in both cultivars starting at $10 \mathrm{dpi}$ (Figures 3C,D). Transcript accumulation of the TRI12 gene also initiated at $2 \mathrm{dpi}$, peaked at $4 \mathrm{dpi}$ and gradually decreased at $7 \mathrm{dpi}$ in most of the strains (Figures 3E,F). Based on the qRT-PCR data, F. graminearum 3-ADON strains showed a higher level of TRI gene expression compared to the other strains for genes FPP, TRI3, TRI4, TRI6, TRI8, TRI12, and TRI101, at most time points except for TRI5 gene in MR cultivar and TRI9 gene. In TRI5 gene, 3-ADON1 and 15-ADON2 strains showed higher levels of expression than other F. graminearum strains in MR cultivar Carberry. The level of gene expression in 15ADON and NIV producing strains showed no specific pattern of higher or lower expression. In some genes and time points the level of transcript accumulation was higher in 15-ADON strains and lower in NIV strains and vice versa. Among the analyzed genes, the highest abundance of transcripts was observed for TRI4 and TRI12 genes for all the examined strains (Figures 1E,F and $3 \mathrm{E}, \mathrm{F})$. Our data showed that relative expression of TRI genes was significantly higher in wheat cultivar Carberry (MR) compared with Roblin (S).

The FHB DS was analyzed at 7 and 14 dpi, terminal FDK and DON/NIV content were analyzed at 14 dpi. When FHB DS was considered, there were significant differences between the cultivars and among the strains. The two-way interaction cultivar*strain was significantly different (Table 1). The highest FHB DS was shown by cultivar Roblin inoculated by 3-ADON strains followed by $15-\mathrm{ADON}$ and NIV strains. FHB DS caused by 3-ADON strains was significantly different from the 15ADON producing strains and NIV strains. A similar trend was observed in the MR cultivar Carberry, however, the FHB symptom development was slower than in cultivar Roblin which is highly susceptible to FHB (Figures 4 A,B). The percentage of FDK was higher in cultivar Roblin than in cultivar Carberry (Figures 5A,B). Similarly, a higher total DON content was observed in cultivar Roblin inoculated with 3-ADON strains than the 15-ADON strains (Figures 5A,B). Cultivars inoculated with 3-ADON strains showed higher levels of FDK and DON content than 15-ADON strains. NIV producing strains showed the lowest FDK percentage and toxin contamination.

\section{DISCUSSION}

The objective of this study was to identify the potential chemotype-specific gene expression patterns of the TRI genes during wheat- F. graminearum infection and colonization. The expression of most TRI genes required for trichothecene production in F. graminearum were strongly induced at early time points after infection (i.e., 2-4 dpi) and the expression levels gradually decreased at $7 \mathrm{dpi}$. Also 3 -ADON producing strains showed a comparatively higher level of gene expression than 15ADON and NIV producing strains, confirming their ability to produce higher amounts of toxin in infected wheat kernels.

Deoxynivalenol biosynthesis related gene expression profiling indicated that, the expression of most TRI genes were initiated at $2 \mathrm{dpi}$. This shows that a high level of TRI transcript accumulation is essential for initiating the biosynthetic pathway of DON or NIV during wheat infection and colonization. The early expression patterns of five TRI genes along with FPP gene (TRI4, TRI5, TRI6, TRI8 and TRI3) strongly suggested that TRI6 gene which encodes a transcriptional regulator, positively regulates the expression of other TRI genes in the DON biosynthesis pathway. Similar observations have reported by Lee et al. (2014) in liquid culture media. The level of TRI gene expression was significantly different among the three chemotypes analyzed. The level of expression of most of the examined genes was higher in $3-\mathrm{ADON}$ producing strains in both cultivars compared to the $15-\mathrm{ADON}$ producing strains and NIV producing strains. It has been reported that 3-ADON strains produce more trichothecenes than 15-ADON and NIV strains (Ward et al., 2008). Therefore, the higher levels of expression of trichothecene biosynthesis related genes in 3-ADON producing strains during colonization may mediate the production of high amounts of toxins. According to the total DON content at $14 \mathrm{dpi}$, kernels infected with 3-ADON strains showed higher total DON content than the 15-ADON strains in both cultivars. Also, in this study the level of transcript accumulation of TRI4 gene was comparatively higher than other genes (except for TRI12 gene). The TRI4 gene regulates multiple steps (four steps) in the trichothecene biosynthesis pathway (McCormick et al., 2006). The accumulation of TRI4 transcripts in higher amounts could be explained by the involvement of this gene in multiple steps during trichothecene production. The level of expression of the TRI12 gene initiated at $2 \mathrm{dpi}$, peaked at $4 \mathrm{dpi}$ and gradually 


\section{TRI6}

A

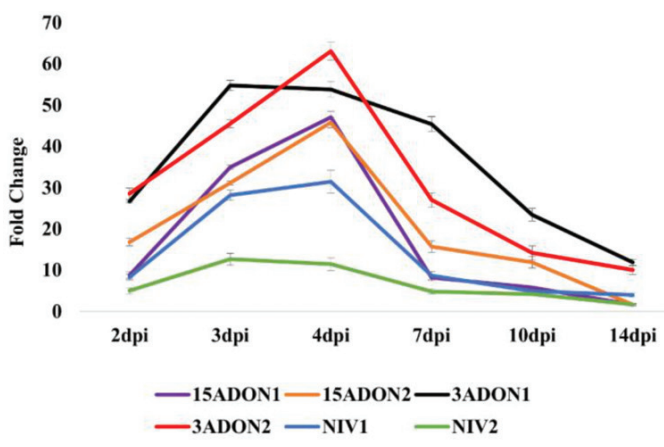

Carberry- MR

\section{TRI8}

C

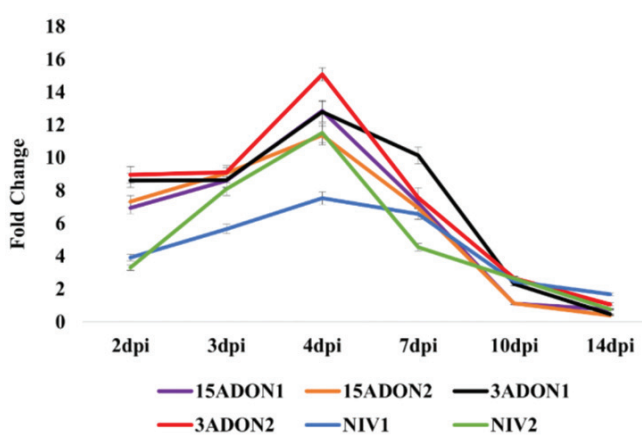

Carberry- MR

\section{TRI101}

E

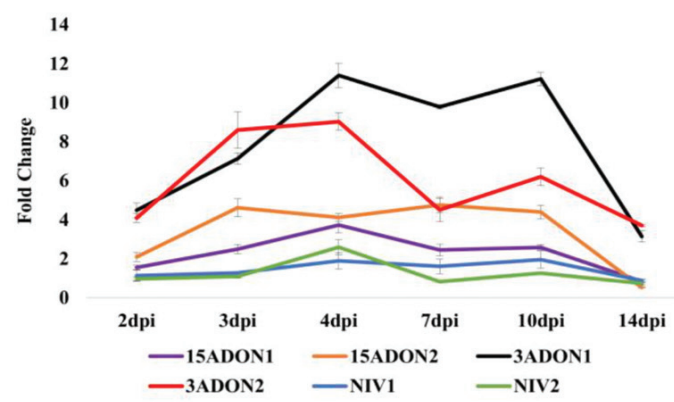

Carberry- MR
B

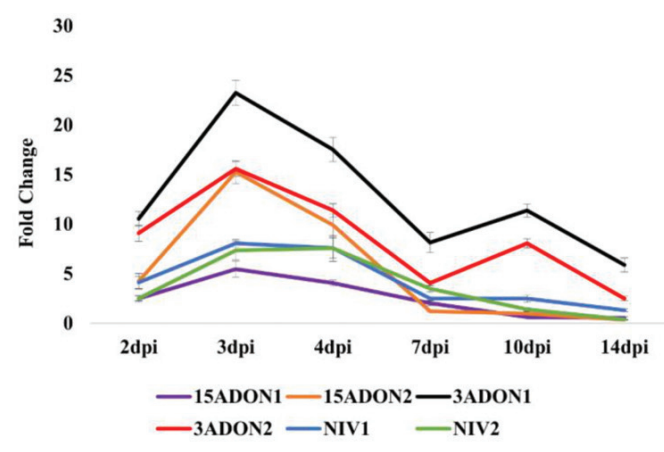

Roblin- S

D

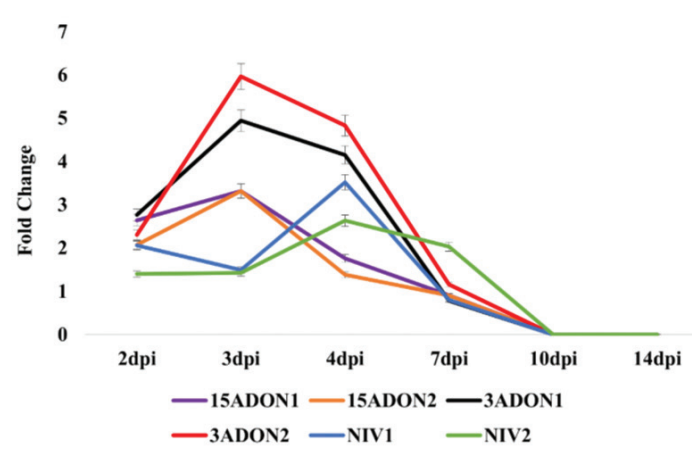

Roblin- S

F

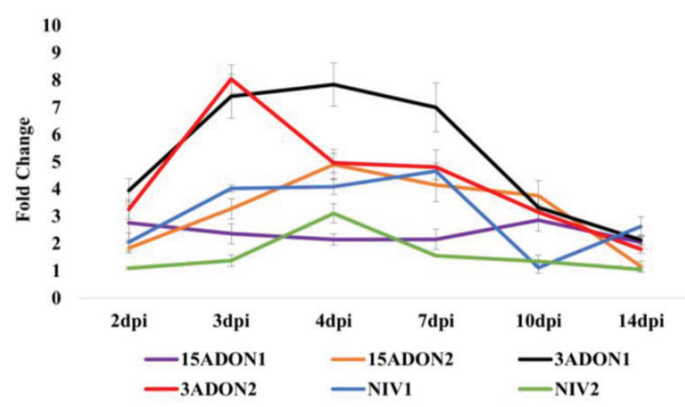

Roblin- S

FIGURE 2 | Fold change in transcript levels of transcription factor TRI6 gene in cultivar Carberry (A) and cultivar Roblin (B), trichothecene 3-O-esterase (TRI8) gene in cultivar Carberry (C) and cultivar Roblin (D) and trichothecene 3-O-acetyltransferase (TRI101) gene in cultivar Carberry (E) and cultivar Roblin (F). 


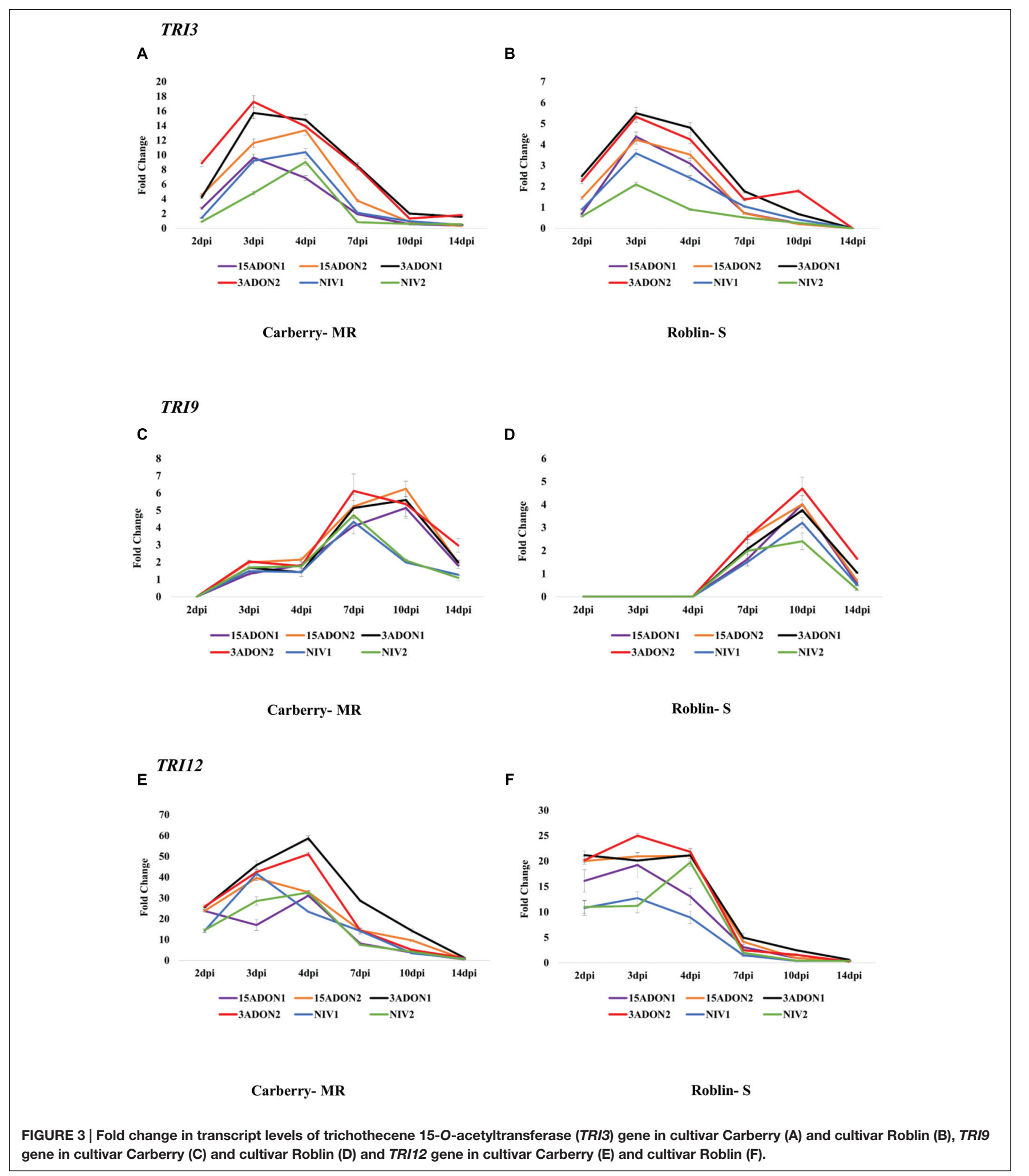

decreased at $7 \mathrm{dpi}$, which was similar to the other analyzed TRI genes in the present study. It has been reported that TRI12 gene encodes for trichothecenes efflux pump, which gives selfprotection for the fungus from the produced trichothecenes
(Alexander et al., 1999). Therefore, coherent gene expression patterns of TR112 genes with other analyzed TRI genes further supports the role of TRI12 gene as a self-protector against the produced trichothecenes. 
TABLE 1 | Analysis of variance (ANOVA) table for cultivar, strain and their interaction for Fusarium head blight disease severity at 7 and 14 days post-inoculation.

\begin{tabular}{llcccr}
\hline $\begin{array}{l}\text { Days } \\
\text { post-inoculation }\end{array}$ & Source & DF & MS & F value & Pr $>$ F \\
\hline 7 dpi & & & & & \\
& Cultivar & 1 & 34556 & 694.69 & $<0.0001$ \\
& Strain & 5 & 1858.88 & 37.37 & $<0.0001$ \\
& Cultivar*Strain & 5 & 478.35 & 9.62 & 0.0001 \\
& Rep & 14 & 60.10 & 1.20 & 0.2806 \\
& Error & 154 & 49.74 & & \\
& Cultivar & 1 & 59405 & 585.26 & $<0.0001$ \\
& Strain & 5 & 4323.23 & 42.59 & $<0.0001$ \\
& Cultivar*Strain & 5 & 2804.13 & 5.53 & 0.0001 \\
& Rep & 14 & 58.06 & 0.57 & 0.8836 \\
& Error & 154 & 101.5 & & \\
\hline
\end{tabular}

The level of expression of TRI genes was significantly higher in the MR cultivar Carberry than in the S cultivar Roblin. Similar results have been reported in other studies (Boddu et al., 2007;
Brown et al., 2011; Hallen-Adams et al., 2011). Still there is no clear reason to explain the higher levels of expression in trichothecene biosynthesis genes in MR cultivar compared to the $S$ cultivar. However, when we analyzed the total DON content at 14 dpi it was higher in the susceptible cultivar Roblin than in the MR cultivar Carberry. DON is a virulence factor in wheat, necessary for the spread of the fungus beyond the point of infection (Proctor et al., 1995; Bai et al., 2002; Audenaert et al., 2013). The above contrary observations between the trichothecene biosynthesis gene expression and terminal DON content in MR and S cultivars can be explained as follows: F. graminearum enters the plant either through inoculation or natural infection; in the first stage, it grows biotrophically at the point of infection and start producing DON (Audenaert et al., 2013). Then the fungus becomes more aggressive and attempts to grow into adjacent spikelets. However, the resistance mechanisms in $\mathrm{R}$ or MR cultivars prevent the fungus invasion from the point of infection. In order to overcome the resistance and spread further from the point of infection, fungus increases DON production as DON acts as a virulence factor in wheat.
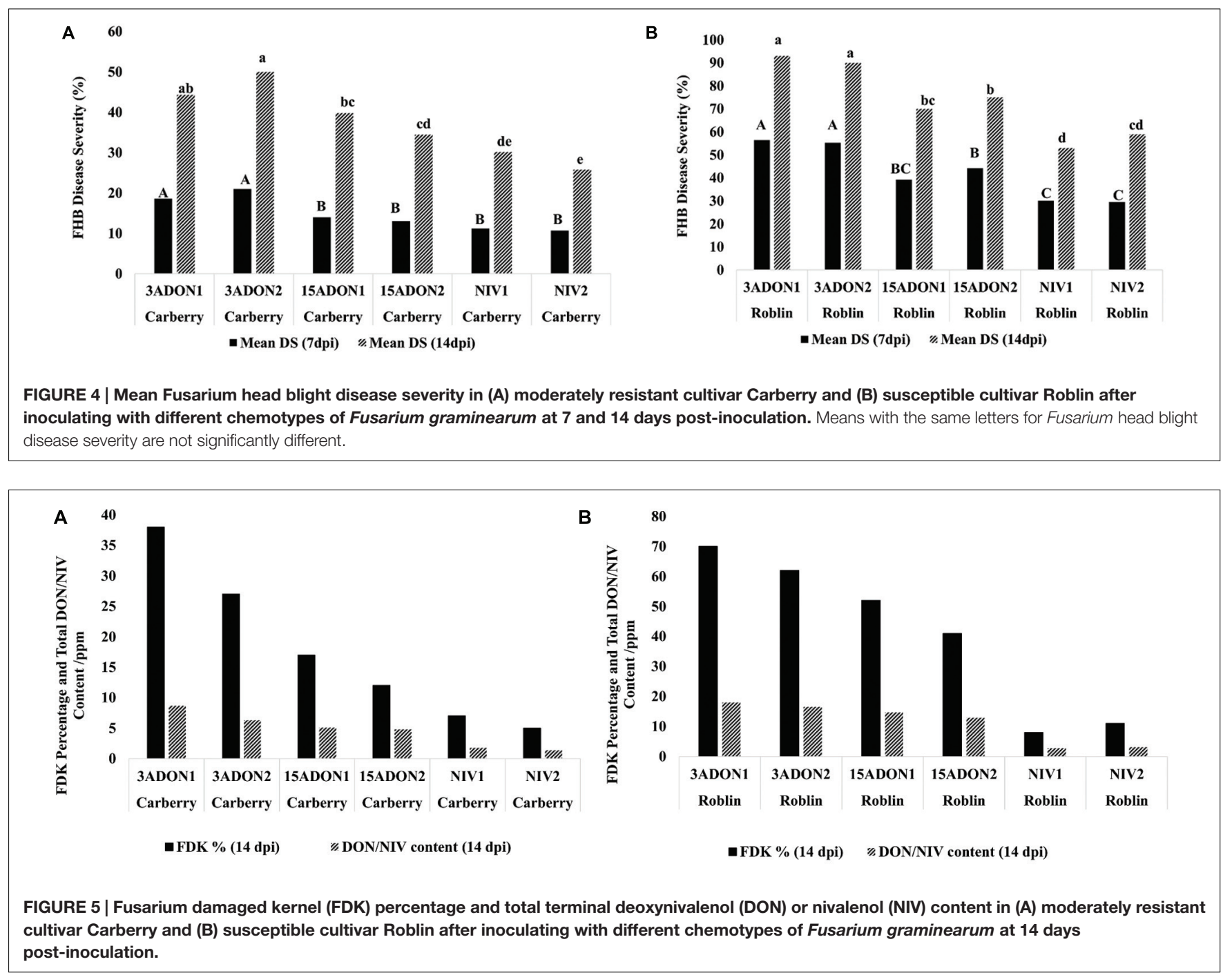
Finally, to increase the DON production, the fungus increases the level of expression of DON biosynthesis related genes in $\mathrm{R}$ or MR cultivars compared to $\mathrm{S}$ cultivars. Investigations are in progress to further understand the reasons for higher levels of TRI gene expression in MR cultivars than in S cultivars.

Although the level of expression of DON biosynthetic genes were higher in the MR cultivar than in the $S$ cultivar the final DON content is higher in the $S$ cultivar. It has been reported that during Fusarium infection there is a broad expression of genes related to the DON detoxification process (Muhovski et al., 2012). This may explain the low levels of DON contamination in MR cultivar compared to S cultivar. Gene expression studies have shown that the expression of DON detoxification transcripts such as UDP-glycosyltransferase family (UGTs), CYP450s, ABC transporters and multidrug resistance-associated protein (MRP) were more highly abundant in FHB resistant cultivars than in susceptible cultivars during Fusarium infection (Muhovski et al., 2012; Al-Taweel et al., 2014; Kosaka et al., 2015). Therefore, it can be hypothesized that, although the level of TRI gene expression is higher in the MR cultivar, the resistance mechanisms within the cultivar can more efficiently detoxify the produced DON than the susceptible cultivar.

This study provides evidence on the chemotype specific gene expression patterns in the DON biosynthesis pathway during wheat infection and colonization. The results from this study indicated that 3 -ADON producing strains showed higher levels of gene expression compared to 15 -ADON and NIV producing strains. However, use of only two strains

\section{REFERENCES}

Alexander, N. J., McCormick, S. P., and Hohn, T. M. (1999). TRI12, a trichothecene efflux pump from Fusarium sporotrichioides: gene isolation and expression in yeast. Mol. Gen. Genet. 261, 977-984. doi:10.1007/s004380051046

Alexander, N. J., McCormick, S. P., Waalwijk, C., van der Lee, T., and Proctor, R. H. (2011). The genetic basis for 3-ADON and 15-ADON trichothecene chemotypes in Fusarium graminearum. Fungal Genet. Biol. 48, 485-495. doi: 10.1016/j.fgb.2011.01.003

Alexander, N. J., Proctor, R. H., and McCormick, S. P. (2009). Genes, gene clusters, and biosynthesis of trichothecenes and fumonisins in Fusarium. Toxin Rev. 28, 198-215. doi: 10.1080/15569540903092142

Al-Taweel, K., Fernando, W. G. D., and Brûlé-Babel, A. L. (2014). Transcriptome profiling of wheat differentially expressed genes exposed to different chemotypes of Fusarium graminearum. Theor. Appl. Genet. 127, 1703-1718. doi: 10.1007/s00122-014-2333-8

Audenaert, K., Vanheule, A., Höfte, M., and Haesaert, G. (2013). Deoxynivalenol: a major player in the multifaceted response of Fusarium to its environment. Toxins (Basel). 6, 1-19. doi: 10.3390/toxins6010001

Bai, G. H., Desjardins, A. E., and Plattner, R. D. (2002). Deoxynivalenolnonproducing Fusarium graminearum causes initial infection, but does not cause disease spread in wheat spikes. Mycopathologia 153, 91-98. doi: 10.1023/ A:1014419323550

Bai, G. H., and Shaner, G. (1994). Scab of wheat: prospect for control. Plant Dis. 78, 760-766. doi: 10.1094/PD-78-0760

Boddu, J., Cho, S., and Muehlbauer, G. J. (2007). Transcriptome analysis of trichothecene-induced gene expression in barley. Mol. Plant Microbe Interact. 20, 1364-1375. doi: 10.1094/MPMI-20-11-1364

Brown, D. W., McCormick, S. P., Alexander, N. J., Proctor, R. H., and Desjardins, A. E. (2001). A genetic and biochemical approach to study trichothecene diversity in Fusarium sporotrichioides and Fusarium graminearum. Fungal Genet. Biol. 32, 121-133. doi: 10.1006/fgbi.2001.1256 representing a chemotype may not be sufficient to draw definitive conclusions. Therefore, this study suggests the use of more strains from each chemotype group to gain a more comprehensive understanding of chemotype specific gene expression patterns during F. graminearum infection and colonization.

\section{AUTHOR CONTRIBUTIONS}

CA performed the research, coordinated the experimental part of the project and wrote the manuscript. WF supervised the research project and critically reviewed the manuscript.

\section{FUNDING}

This work was supported by Western Grains Research Foundation (WGRF), National Wheat Improvement Program and Agri-Food Research and Development Initiative Manitoba (ARDI).

\section{ACKNOWLEDGMENTS}

We acknowledge Canadian Wheat Board and University of Manitoba Graduate Fellowship for the financial support provided to CA.

Brown, N. A., Bass, C., Baldwin, T. K., Chen, H., Massot, F., Carion, P. W. C., et al. (2011). Characterisation of the Fusarium graminearum-Wheat floral interaction. J. Pathog. 2011:626345. doi: 10.4061/2011/626345

Cuthbert, P. A., Somers, D. J., Tomas, J., Clotier, S., and Brule-Babel, A. (2006). Fine mapping Fhb1, a major gene controlling fusarium head blight resistance in bread wheat (Triticum aestivum L.). Theor. Appl. Genet. 112, 1465-1472. doi: 10.1007/s00122-006-0249-7

Desjardins, A. E., Hohn, T. M., and McCormick, S. P. (1993). Trichothecene biosynthesis in Fusarium species: chemistry, genetics, and significance. Microbiol. Rev. 57, 595-604. doi: 10.1128/CMR.16.3.497

Diamond, M., Reape, T. J., Rocha, O., Doyle, S. M., Kacprzyk, J., Doohan, F. M., et al. (2013). The Fusarium mycotoxin deoxynivalenol can inhibit plant apoptosis-like programmed cell death. PLOS ONE 8:e69542. doi: 10.1371/journal.pone.0069542

Foroud, N. A., and Eudes, F. (2009). Trichothecenes in cereal grains. Int. J. Mol. Sci. 10, 147-173. doi: 10.3390/ijms10010147

Gale, L. R., Bryant, J. D., Calvo, S., Giese, H., Katan, T., O’Donnell, K., et al. (2005). Chromosome complement of the fungal plant pathogen Fusarium graminearum based on genetic and physical mapping and cytological observations. Genetics 171, 985-1001. doi: 10.1534/genetics.105. 044842

Gardiner, D. M., Kazan, K., and Manners, J. M. (2009). Nutrient profiling reveals potent inducers of trichothecene biosynthesis in Fusarium graminearum. Fungal Genet. Biol. 46, 604-613. doi: 10.1016/j.fgb.2009.04.004

Goswami, R. S., and Kistler, H. C. (2005). Pathogenicity and in planta mycotoxin accumulation among members of the Fusarium graminearum species complex on wheat and rice. Phytopathology 95, 1397-1404. doi: 10.1094/PHYTO-951397

Hallen-Adams, H. E., Wenner, N., Kuldau, G. A., and Trail, F. (2011). Deoxynivalenol biosynthesis- related gene expression during wheat kernel colonization by Fusarium graminearum. Phytopathology 101, 1091-1096. doi: 10.1094/PHYTO-01-11-0023 
Hohn, T. M., and Beremand, P. D. (1989). Isolation and nucleotide sequence of a sesquiterpene cyclase gene from the trichothecene-producing fungus Fusarium sporotrichioides. Gene 79, 131-138. doi: 10.1016/0378-1119(89) 90098-X

Kim, H., and Yun, S. (2011). Evaluation of potential reference genes for quantitative rt-pcr analysis in Fusarium graminearum under different culture conditions. Plant Pathol. J. 27, 301-309. doi: 10.5423/PPJ.2011.27.4.301

Kosaka, A., Manickavelu, A., Kajihara, D., Nakagawa, H., and Ban, T. (2015). Altered gene expression profiles of wheat genotypes against Fusarium head blight. Toxins (Basel). 7, 604-620. doi: 10.3390/toxins 7020604

Lee, T., Han, Y., Kim, K.-H., Yun, S.-W., and Lee, Y. W. (2002). Tri13 and Tri7 determine deoxynivalenol- and nivalenol-producing chemotypes of Gibberella zeae. Appl. Environ. Microbiol. 68, 2148-2154. doi: 10.1128/AEM.68.5.21482154.2002

Lee, T., Lee, S. H., Shin, J. Y., Kim, H. K., Yun, S. H., Kim, H. Y., et al. (2014). Comparison of trichothecene biosynthetic gene expression between Fusarium graminearum and Fusarium asiaticum. Plant Pathol. J. 30, 33-42. doi: 10.5423/PPJ.OA.11.2013.0107

Leslie, J. F., and Summerell, B. A. (2006). The Fusarium Laboratory Manual. Ames, IA: Blackwell Publishing, 6-7. doi: 10.1002/9780470278376

McCormick, S. P., and Alexander, N. J. (2002). Fusarium Tri8 encodes a trichothecene C-3 esterase. Appl. Environ. Microbiol. 68, 2959-2964. doi: 10.1128/AEM.68.6.2959

McCormick, S. P., Alexander, N. J., and Proctor, R. H. (2006). Fusarium Tri4 encodes a multifunctional oxygenase required for trichothecene biosynthesis. Can. J. Microbiol. 52, 636-642. doi: 10.1139/w06-011

McMullen, M., Jones, R., Gallenberg, D., and America, S. (1997). Scab of wheat and barley: a re-emerging disease of devastating impact. Plant Dis. 81, 1340-1348. doi: 10.1094/PDIS.1997.81.12.1340

Merhej, J., Richard-Forget, F., and Barreau, C. (2011). Regulation of trichothecene biosynthesis in Fusarium: recent advances and new insights. Appl. Microbiol. Biotechnol. 91, 519-528. doi: 10.1007/s00253-011-3397-x

Muhovski, Y., Batoko, H., and Jacquemin, J. M. (2012). Identification, characterization and mapping of differentially expressed genes in a winter wheat cultivar (Centenaire) resistant to Fusarium graminearum infection. Mol. Biol. Rep. 39, 9583-9600. doi: 10.1007/s11033-012-1823-5

Pfaffl, M. W., Horgan, G. W., and Dempfle, L. (2002). Relative expression software tool (REST) for g Fusarium Tri4 encodes a multifunctional oxygenase required for trichothecene biosynthesis roup-wise comparison and statistical analysis of relative expression results in real-time PCR. Nucleic Acids Res. 30:e36. doi: 10.1093/nar/30.9.e36

Proctor, R. H., Hohn, T. M., and McCormick, S. P. (1995). Reduced virulence of Gibberella zeae caused by disruption of a trichothecene toxin biosynthetic gene. Mol. Plant Microbe Interact. 8, 593-601. doi: 10.1094/MPMI8-0593

Sobrova, P., Adam, V., Vasatkova, A., Beklova, M., Zeman, L., and Kizek, R. (2010). Deoxynivalenol and its toxicity. Interdiscip. Toxicol. 3, 94-99. doi: 10.2478/v10102-010-0019-x

Stack, R., and McMullen, M. (1995). A Visual Scale to Estimate Severity of Fusarium Head Blight of Wheat. http://www.ag.ndsu.edu/pubs/plantsci/smgrai ns/pp1095.pdf

Tittlemier, S. A., Gaba, D., and Chan, J. M. (2013). Monitoring of Fusarium trichothecenes in Canadian cereal grain shipments from 2010 to 2012. J. Agric. Food Chem. 61, 7412-7418. doi: 10.1021/jf4019257

Wang, J. -H., Ndoye, M., Zhang, J. -B., Li, H. -P., and Liao, Y. -C. (2011). Population Structure and Genetic Diversity of the Fusarium graminearum Species Complex. Toxins (Basel). 3, 1020-1037. doi: 10.3390/toxins3081020

Ward, T. J., Clear, R. M., Rooney, A. P., O’Donnell, K., Gaba, D., Patrick, S., et al. (2008). An adaptive evolutionary shift in Fusarium head blight pathogen populations is driving the rapid spread of more toxigenic Fusarium graminearum in North America. Fungal Genet. Biol. 45, 473-484. doi: 10.1016/j.fgb.2007.10.003

Yazar, S., and Omurtag, G. Z. (2008). Fumonisins, trichothecenes and zearalenone in cereals. Int. J. Mol. Sci. 9, 2062-2090. doi: 10.3390/ijms9112062

Zhang, Y.-J., Yu, J.-J., Zhang, Y.-N., Zhang, X., Cheng, C.-J., Wang, J.-X., et al. (2009). Effect of carbendazim resistance on trichothecene production and aggressiveness of Fusarium graminearum. Mol. Plant Microbe Interact. 22, 1143-1150. doi: 10.1094/MPMI-22-9-1143

Conflict of Interest Statement: The authors declare that the research was conducted in the absence of any commercial or financial relationships that could be construed as a potential conflict of interest.

Copyright (c) 2016 Amarasinghe and Fernando. This is an open-access article distributed under the terms of the Creative Commons Attribution License (CC BY). The use, distribution or reproduction in other forums is permitted, provided the original author(s) or licensor are credited and that the original publication in this journal is cited, in accordance with accepted academic practice. No use, distribution or reproduction is permitted which does not comply with these terms. 